\title{
DHM simulation in virtual environments: a case-study on control room design
}

\author{
Zamberlan, $\mathrm{M}^{\mathrm{a},{ }^{*}}$, Santos, $\mathrm{V}^{\mathrm{b}}$, Streit, $\mathrm{P}^{\mathrm{a}}$, Oliveira, $\mathrm{J}^{\mathrm{a}}$, Cury, $\mathrm{R}^{\mathrm{a}}$, Negri, $\mathrm{T}^{\mathrm{a}}$, Pastura, $\mathrm{F}^{\mathrm{a}}$, Guimarães, $\mathrm{C}^{\mathrm{a}}$ and \\ Cid, $\mathrm{G}^{\mathrm{a}}$ \\ a Instituto Nacional de Tecnologia, INT. Avenida Venezuela, 82, Rio de Janeiro, RJ. Brasil - 20081-312 \\ ${ }^{b}$ Pontifícia Universidade Católica do Rio de Janeiro - PUC-Rio. Rua Marquês de São Vicente, 225, Gávea, Rio \\ de Janeiro, RJ - Brasil - 22451-900
}

\begin{abstract}
This paper will present the workflow developed for the application of serious games in the design of complex cooperative work settings. The project was based on ergonomic studies and development of a control room among participative design process. Our main concerns were the 3D human virtual representation acquired from 3D scanning, human interaction, workspace layout and equipment designed considering ergonomics standards. Using Unity3D platform to design the virtual environment, the virtual human model can be controlled by users on dynamic scenario in order to evaluate the new work settings and simulate work activities. The results obtained showed that this virtual technology can drastically change the design process by improving the level of interaction between final users and, managers and human factors team.
\end{abstract}

Keywords: digital human model, simulation, virtual environment, motion capture

\section{Introduction}

The research in serious games has been a focus issue for Government and corporate organizations, applied on training, simulation and education. There is a clear need for considering new frameworks, theories, methods and design strategies for making serious games applications and virtual world technologies more effective and useful as part of education, health and training. Virtual simulation has been used in Ergonomics for the design of control centers, transport design and product evaluation. (SANTOS, V., et al. 2009, SANTOS,V. et al. 2008, GUIMARÃES, C. P. et al. 2010).

The aim of this paper is to discuss the use of serious games applied in a control room design and how virtual reality and game engines may improve the workflow for the design of complex cooperative situations.

The project's context was to find a way to improve and accelerate participative design process for a new emergency control room. Due to a low level of availability and dispersion of stakeholders, it was decided to explore how serious games may improve the participative design. From a human factors point of view, there are strong constraints because: (1) future users must not only "see" their future worksettings in a virtual space but also be able to project their future actions in order to anticipate the pros and cons of the new situation,(2) the workflow must be very dynamic in order to maintain a momentum for the participative process. The different versions of serious games must be synchronized with the organizational process. With modern game engines, this constraint may be easily satisfied; and (3) reusability of virtual material (both objects and movements) is also an important issue.

Another important aspect regarding this specific project is to reproduce cognitive features. In other words, one user should be able to visualize the others' intentions, processes and actions trajectories in order to make decisions, such as where someone is looking at, their reactions to adversities, "volume" of communication, dispersion and others.

In this context, one of the main concerns was the 3D human virtual representation. Other important issues studied were human interaction, workspace

\footnotetext{
* Venétia Santos. E-mail: ergon@ergonprojetos.com.br
} 
layout and equipment including their GUI (Graphic User Interface).

\section{Materials and methods}

The project's stages consisted in (1) gathering information of existing control rooms and of operational personnel in different shifts by a multidisciplinary team that evaluated ergonomics and architectural aspects, personnel data (interviews, filming and analyzing activities, motion capture, 3D human scanning), internal communication, displacement and quantification of work volume, in order to design alternatives, (2) developing these alternatives in walk-around 3D virtual scenarios, so their evaluation is more effective, (3) developing a simulator from the chosen alternative.

The development of the simulator were segmented in a couple of stages: (1) building the 3D environment based on 2D CAD representation of the chosen proposal, (2) implementing furniture and workstations from an existing database with ergonomic recommendations and the application of Brazilian and International standards, (3) development of equipment and instruments' 3D models, (4) development of the DHMs based on 3D scanning and motion capture technologies and (5) implementation in the game engine with scripts and other setups.

Each 2D CAD proposal (Figure 1) was transformed into $3 \mathrm{D}$ virtual models, where later in Unity3D were applied interactive elements such as doors and windows, external scenarios, materials and textures, so that the environment is as realistic as possible, providing the user a more lifelike experience (Figure 2).

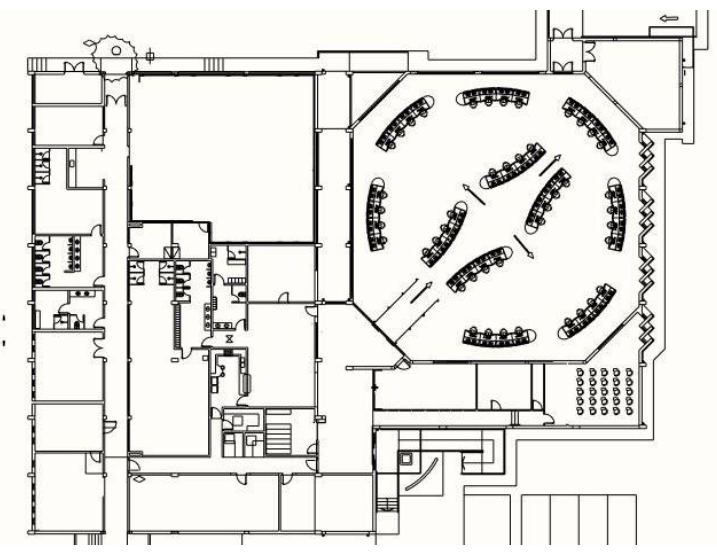

Fig. 1. 2D CAD floorplan

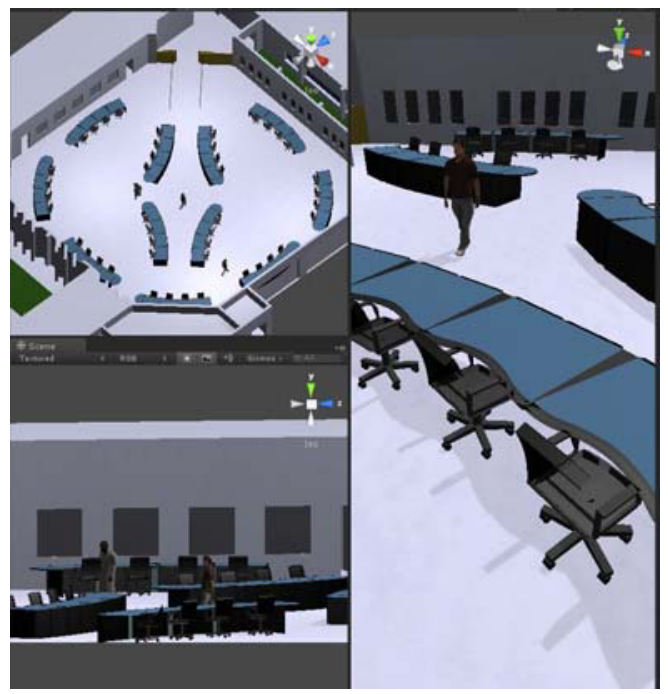

Fig. 2. 3D environment in Unity3D

Furniture and workstations present in the virtual laboratory are reproduced based on ergonomics recommendations and standards, modeled in SketchUp software and implemented in a database including 3D equipment and other instruments (Figure 3). The 3D database allows direct exporting to the game platform in *.FBX format. The software allows the segmentation of interactive components in groups, which in Unity3D can be easily set up by its hierarchy characteristic. It also exports their textures in *.JPEG, which are reproduced using their own pictures as textures (Figure 4), providing clearer understanding and faster recognition of each equipment by their respective users.

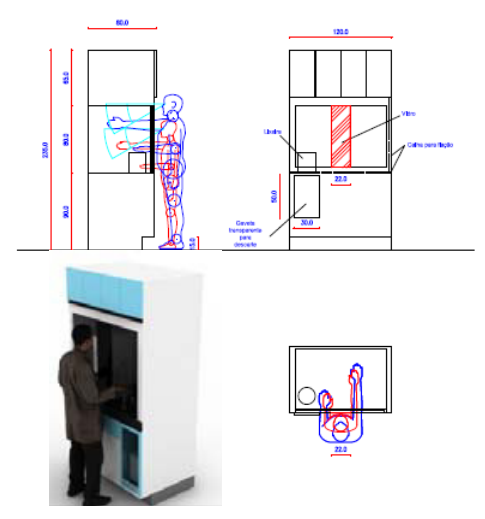

Fig. 3. Furniture recommendations 


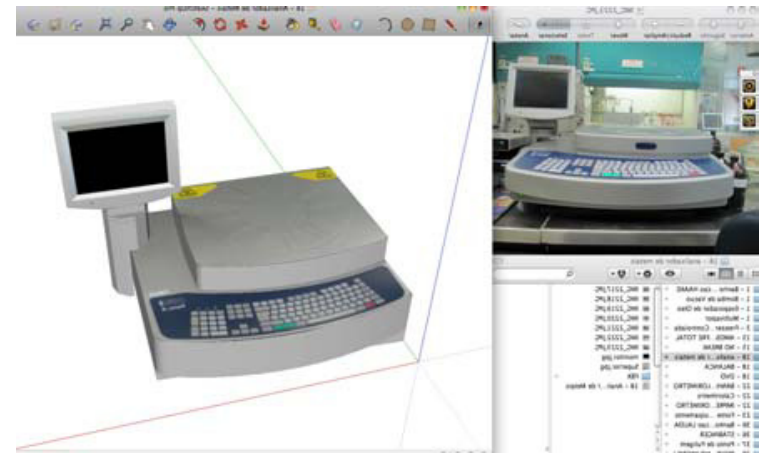

Fig. 4. Equipment 3D database.

DHM used in the simulation have been obtained starting from high resolution DHM (more than one million vertex) obtained by $3 \mathrm{D}$ scanning of employees using "Cyberware Whole Body Color 3D Scanner" and "Cyberware Head and Face Color 3D Scanner".

To import the avatars into the virtual work environment in the Unity3D, their meshes must be seriously reduced. The high poly scan is imported into a 3D software to make a manual re-topology doing this process manually allows us to control edge-flow of each model, therefore, keep expression details enhanced by subdividing the T-zone - eyes, nose and mouth. For the body, it's important to maintain articulations also with higher poly count to avoid deformations in the low poly mesh and keep the movements fluid.

To generate a planar texture which can be edited in graphic software, the process of retopology must consider the following step of marking the loops for UV mapping, so it can be opened properly (Figure 5).

To apply the texture in the low poly model, there are two approaches: (1) capturing photographs during the scanning process in order to have several pictures in different angles, which provides better resolution (Figure 6) and (2) rendering the high poly model, which enables sectioning the model in order to have hidden surface images, such as armpits, inner legs, top and bottom views.

The result of such a process is a realistic virtual representation of the user (low poly and detailed mesh of the user) that respects with high fidelity the morphology of the person that was scanned (Figure 7).

Most of the animation were acquired using "Moven" motion capture suite. The motion capture sessions took place in the laboratory with personnel performing daily activities. The motion capture files were exported to an animation format recognized by 3D Max (*.bvh format), then were edited and imported into the digital human models .

In this process, the low poly model (*.obj format) is imported into 3D Max, where the biped structure is placed, correctly skinned to respond to each bone movement and exported to MotionBuilder.

Inside MotionBuilder, the biped structure is characterized to follow the bones naming conventions and the motion capture files are imported, animations are then divided into full animation and cycle animations, as walk and idle, animations that play on loop, avoiding that the animation loading takes too much time.

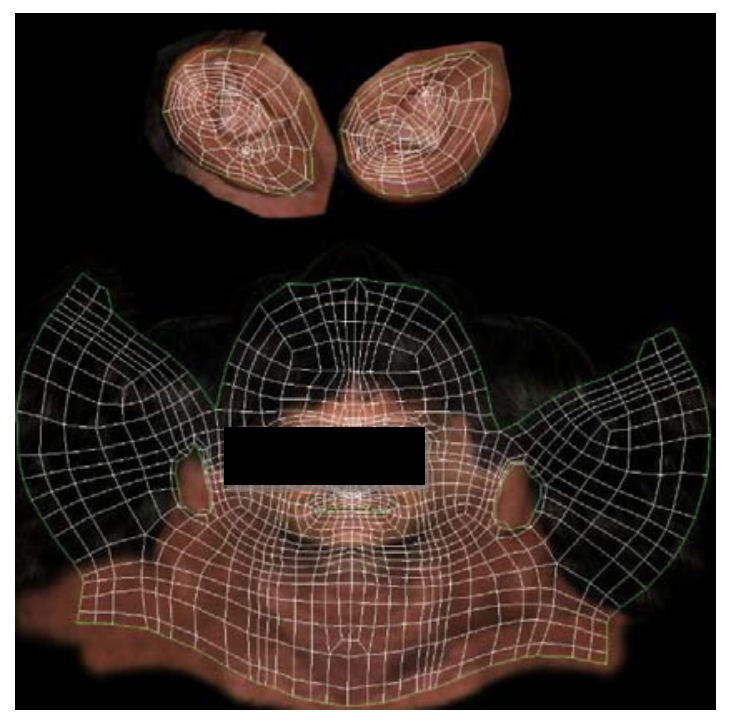

Fig. 5. Planar texture and UV map.

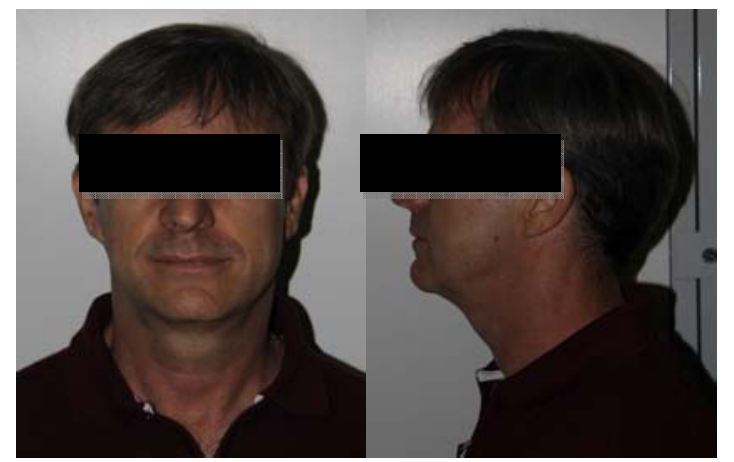

Fig. 6. Example of captured pictures during the scanning process. 

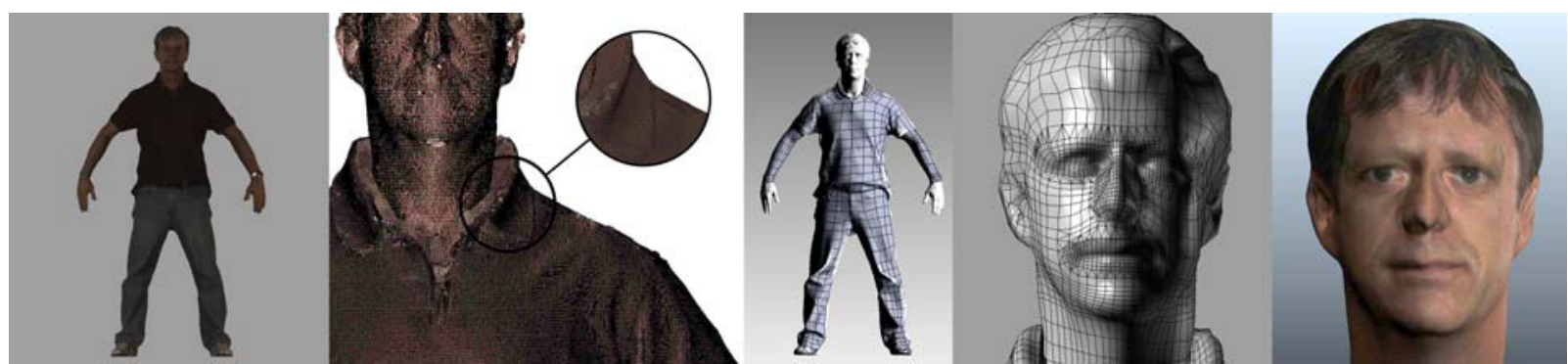

Fig. 7. Retopology and painting process/result.

After all animations are correctly set to its character, the animation is plotted to the skeleton and the DHM is sent back to 3D Max were the empty skeleton animation will be updated for those sent by MotionBuilder. At this time the file is already set to be exported to Unity with its correct media and animation.

\section{Results}

One of the simulator's goal is to allow multiple users to interact with each other using their own avatars. It will provide the team the ability to train activities, learn more about how their colleagues proceed and train new personnel.

In the final steps of the development of the project, the network function is set among all the $3 \mathrm{D}$ models. (Figure 8). This setup involves: (1) scripting the objects (most of them are in java language - e.g. interactive doors, drawers, equipments, picking up and dropping objects), (2) scripting the scene (menus, cameras, interactive cameras, networking, illumination, layout, possibility to enable or disable a trail renderer in order to map users paths across the laboratory). All these components are brought to the users through a friendly GUI (Graphic User Interface), which makes the simulator as intuitive as possible, so the users can focus on the activities and not on how to work with the engine.

\section{Conclusion}

Nowadays, industrial projects are developed with the use of 3D software engines instead of 2D tools, allowing the main focus to be human labor and not only the project itself. Allying it to the study of social interactions at work helps to project better environments.
The use of virtual environments gives the possibility to discuss, change, create and deliver a better result as it is more graphic and visual for non architects and designers professionals, to understand and discuss the new layout of the work space where can be chosen a better design alternative, optimize the interfaces, integrate countless projects and a great number of professionals involved.

Transparency of the future project allows adjustments and error recovery throughout the design process. Projects become more robust, since the scenarios and future activities may be simulated and also the risks involved studied.

These simulations may be used to evaluate technology, industrial safety and/or human performance. One may map process risks, ergonomic and architectural problems, escape routes, displacement of people in crisis situations, assembly and maintenance problems.

Therefore the conclusion is that virtual simulators of social interactions contribute towards: the activity of designers in the occupation of three-dimensional space; evaluation of possible alternatives; detailing the environment; validation of the future project by users, managers, and others; safety, health and environment evaluation; training of human resources.

Even if character interaction cannot always be done in the virtual space, stakeholders can easily project their knowledge of the working situation in order to assess part of the new working space characteristics improving the participative dimension of the project.

Organizational decisions were taken around these tools; they help people to project themselves in their future working spaces and furthermore it was a great tool to improve the feeling of participation. 


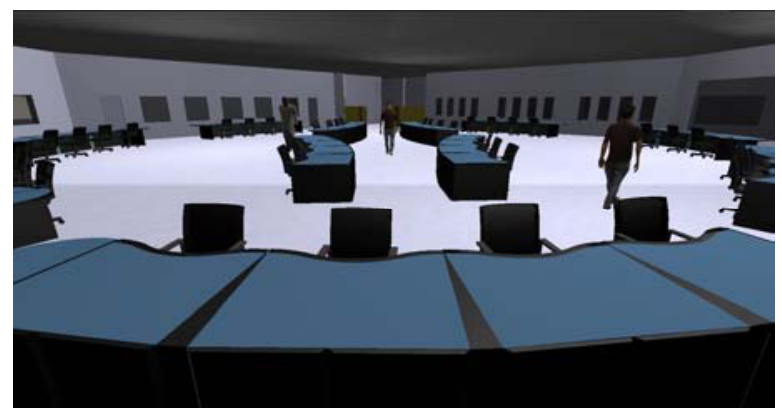

Fig. 8. Control room simulator running.

\section{References}

[1] V. Santos; M.C.P.L. Zamberlan; P.; B. Pavard; P. Streit; J.L. Oliveira; C.P. Guimarães; F.C.H. Pastura, Social Interaction Simulators: Serious games for the design of complex sociotechnical systems. DHM 2011 - First International Symposium on Digital Human Modeling, UCBL - Université Claude-Bernard Lyon, 2011

[2] V. Santos; M.C.P.L. Zamberlan; P.; B. Pavard, Confiabilidade Humana e Projeto Ergonômico de Centros de Controle de Processos de Alto Risco, ed., Synergia, Rio de Janeiro, 2009.

[3] V. Santos; C. Guimarães; G. Cid, Simulação Virtual e Ergonomia, XV Congresso Brasileiro de Ergonomia, VI Fórum Brasileiro de Ergonomia, Porto Seguro, 2008.

[4] C. Guimarães; F.C.H. Pastura; B. Pavard; N. Pallamin; G. Cid; V. Santos; M.C.P.L. Zamberlan, Ergonomics Design Tools Based on Human Work Activities, 3D Human Models and Social Interaction Simulation IHX Congress, Miami, 2010 